\title{
Effects of sclerosing agents on head and neck hemangiomas: A systematic review
}

\author{
Rúbia-Teodoro Stuepp ${ }^{1,2}$, Fernanda-Marcello Scotti ${ }^{1,2}$, Gilberto Melo ${ }^{1}$, Etiene-de Andrade Munhoz ${ }^{2}$, Filipe \\ Modolo $^{3}$
}

${ }^{1}$ Postgraduate Program in Dentistry, Federal University of Santa Catarina (UFSC), Florianopolis, Brazil

${ }^{2}$ Department of Dentistry, Federal University of Santa Catarina (UFSC), Florianopolis, Brazil

${ }^{3}$ Department of Pathology, Federal University of Santa Catarina (UFSC), Florianopolis, Brazil

Correspondence:

Department of Dentistry

Center of Health Sciences

Federal University of Santa Catarina

University Campus

Trindade, Florianópolis, 88.040-900

Santa Catarina, Brazil

rubia_ts@hotmail.com

Stuepp RT, Scotti FM, Melo G, Munhoz EA, Modolo F. Effects of sclerosing agents on head and neck hemangiomas: A systematic review. J Clin Exp Dent. 2019;11(11):e1033-44.

http://www.medicinaoral.com/odo/volumenes/v11i11/jcedv11i11p1033.pdf

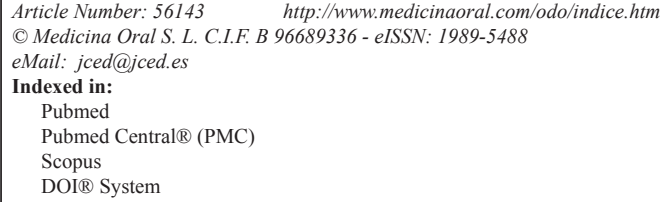

\begin{abstract}
Background: the aim of this study was to systematically review the literature for studies that investigated the effects of sclerosing agents on head and neck hemangiomas.

Material and Methods: clinical trials, cohort studies, and descriptive studies were considered eligible and selected in a two-phase process. Six main electronic databases, in addition to three grey literature databases, were searched. Risk of bias (RoB) was assessed using the "Meta-Analysis of Statistics Assessment and Review Instrument" checklist. From fifty-six considered eligible, five were finally included.

Results: one article were judged at low, one at moderate, and three at high RoB. The sclerosing agents investigated were sodium tetradecyl sulphate $(n=2)$, ethanolamine oleate $(n=1)$, pingyangmycin $(n=1)$ and bleomycin $(n=1)$. Overall, good results were achieved on the treatment of head and neck hemangiomas with intralesional sclerotherapy. Most commonly reported adverse effects included pain, swelling, fever, necrosis, transient facial palsy, and anorexia. Conclusions: considering the limited number of included studies, intralesional sclerotherapy on the management of $\mathrm{HN}$ hemangiomas presented overall good results with minor adverse reactions, especially in regards to smaller lesions.
\end{abstract}

Key words: Sclerotherapy, sclerosing solutions, vascular neoplasms, hemangioma.

\section{Introduction}

Vascular anomalies comprise a heterogeneous group of lesions that have been classified by the International Society for the Study of Vascular Anomalies (ISSVA) into vascular malformations and vascular tumors (1). Hemangioma is a common type of benign vascular tumor that usually affects newborns and infants, although in some cases it might persist into adulthood (2). It should be mentioned that misdiagnosed of vascular tumors and vascular malformations is still recurrent and this can lead to misconduct, therefore, caution should be exercised regarding assessment of these conditions. 
Approximately $10-12 \%$ of children at 1 year of age are affected by this condition (2) and nearly $60 \%$ of hemangiomas are located in the head and neck (HN) region, (3$5)$ specially the face, oral mucosa, lips, and tongue $(2,4)$. Some subsets of hemangioma have been documented. Congenital hemangiomas $(\mathrm{CH})$ are fully formed at birth and according to its clinical course, can be subdivided into two major subgroups: rapidly involuting congenital hemangioma $(\mathrm{RICH})$ and noninvoluting congenital hemangioma $(\mathrm{NICH})(6)$. The first one rapidly regress within 3 to 5 months and the second remains static in their clinical course (7).

Infantile hemangiomas (IH) are the most common benign vascular tumor of childhood and differs from $\mathrm{CH}$ in histologic features and immunophenotype, as well as clinical presentation and behavior (7). At birth, IH often appears as a precursor lesion, such as an area of telangiectasia or small purple area, then increases and become more recognizable. A fast growth occurs at three to five months; thereafter it usually involutes in several year, although abnormal texture, color, or residual fibroadipose tissue might persist on the overlying skin $(3,8)$.

About $50 \%$ of hemangiomas have a complete resolution in 5 years and $70 \%$ in 7 years. Most of the lesions are small and usually no treatment is indicated (9). However, treatment is often indicated for those large lesions that present rapid growth, located in cosmetically areas, or presenting complications, such as ulceration, pain, bleeding, secondary infection, and tissue deformation $(3,4,10)$. Possible psychosocial consequences on the affected child and family should also be considered with regard to treatment decisions (11).

To date, there is yet no consensus on the treatment of hemangiomas. Nonetheless, surgical excision is no longer the first choice (4) since it is related with complications such as bleeding, scarring, organ and tissue dysfunction (12), nerve damage (13), and often results in residual pathology (5). Still, many nonsurgical treatments have been attempted, including systemic propranolol and corticosteroids, interferon- $\alpha$, lasertherapy, embolization, cryotherapy, radiotherapy, intralesional sclerotherapy, among others (10). Sclerosing agents applied intralesionally promotes lesion reduction and sclerosis (13). However, a wide range of sclerosing agents have been documented and its effectiveness and safety remains unclear (13).

The aim of this systematic review (SR) was to investigate the effectiveness and safety of sclerosing agents on $\mathrm{HN}$ hemangiomas and provide to physicians and dental clinicians an evidence-based therapeutic decision-making.

\section{Material and Methods}

A SR protocol was registered at Prospective Register of Systematic Reviews under the registration number CRD 42018100394 (14). The reporting of this SR was based on PRISMA recommendations (15). The acronym PICOS (Population, Intervention, Comparison, Outcome, Studies) was used to formulate the question of this study, of which: P) individuals diagnosed with $\mathrm{HN}$ hemangiomas; I) intralesional sclerotherapy; C) other therapies, placebo, or pre-treatment status; O) lesion size reduction and complications; and S) clinical trials, cohort studies, or case-series with at least 10 participants. Only articles published in Latin Roman alphabet were considered.

The following exclusion criteria were applied: 1) studies that evaluated animals; 2) case-series with less than 10 participants with HN hemangiomas; 3) studies that combined sclerosing agents with other therapies; 4) studies investigating sclerosing therapy for vascular malformations or that did not provide separate data for hemangiomas; 5) studies investigating sclerosing therapy for peri- or intra-orbital vascular tumors; 6) studies in which outcomes for different sclerosing agents or different lesion sites were not reported separately; 7) abstracts, reviews, case-reports, protocols, laboratory research; 8) studies not published in the Latin Roman alphabet; and 9) full-text not available.

Search strategies were elaborated for the following electronic databases: Embase, Latin American and Caribbean Health Sciences (LILACS), PubMed, SCOPUS, The Cochrane Library, and Web of Science. In addition, a grey literature search was conducted on Google Scholar, Open Grey, and ProQuest. All searches were performed from the starting coverage date through May 12, 2019. Detailed search strategies are provided in Table 1, 1 continue.

Furthermore, following the recommendation by Greenhalgh and Peacock (16), reference lists of included studies were hand-searched to assess relevant references. Reference management and removal of duplicates were performed using software (EndNote X7, Thomson Reuters). The selection process was performed in two phases. Phase- 1 was carried out in a web application (Rayyan $\AA$, Qatar Computing Research Institute). Firstly, two blinded reviewers (R.T.S and F.M.S.) screened title and abstracts of all identified studies and discrepancies were resolved by a consensus discussion; if necessary, a third reviewer was involved (G.M.). Thereafter, the same reviewers applied the eligibility criteria to full-text articles; if a consensus was not achieved, the third reviewer was consulted. Studies were included for analysis if minimum inclusion criteria were met.

Two blinded reviewers (R.T.S. and F.M.S.) collected data from included studies; information was then crosschecked to warrant integrity of contents. Gathered data consisted of study characteristics, population characteristics, intervention characteristics, outcome measures, and adjustment factors.

Risk of bias (RoB) was independently assessed by two reviewers (R.T.S. and F.M.S.) using the Joanna Briggs 


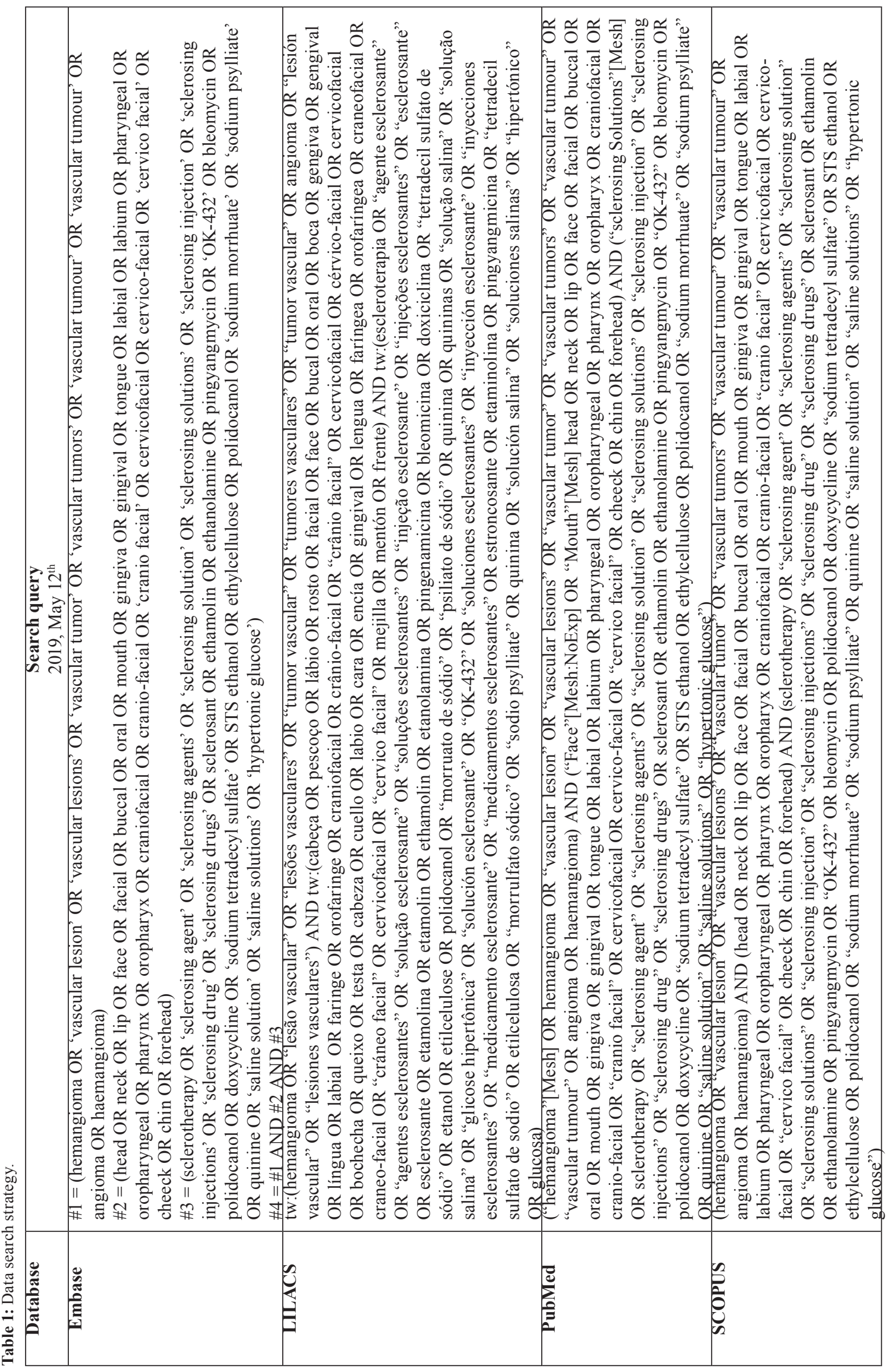




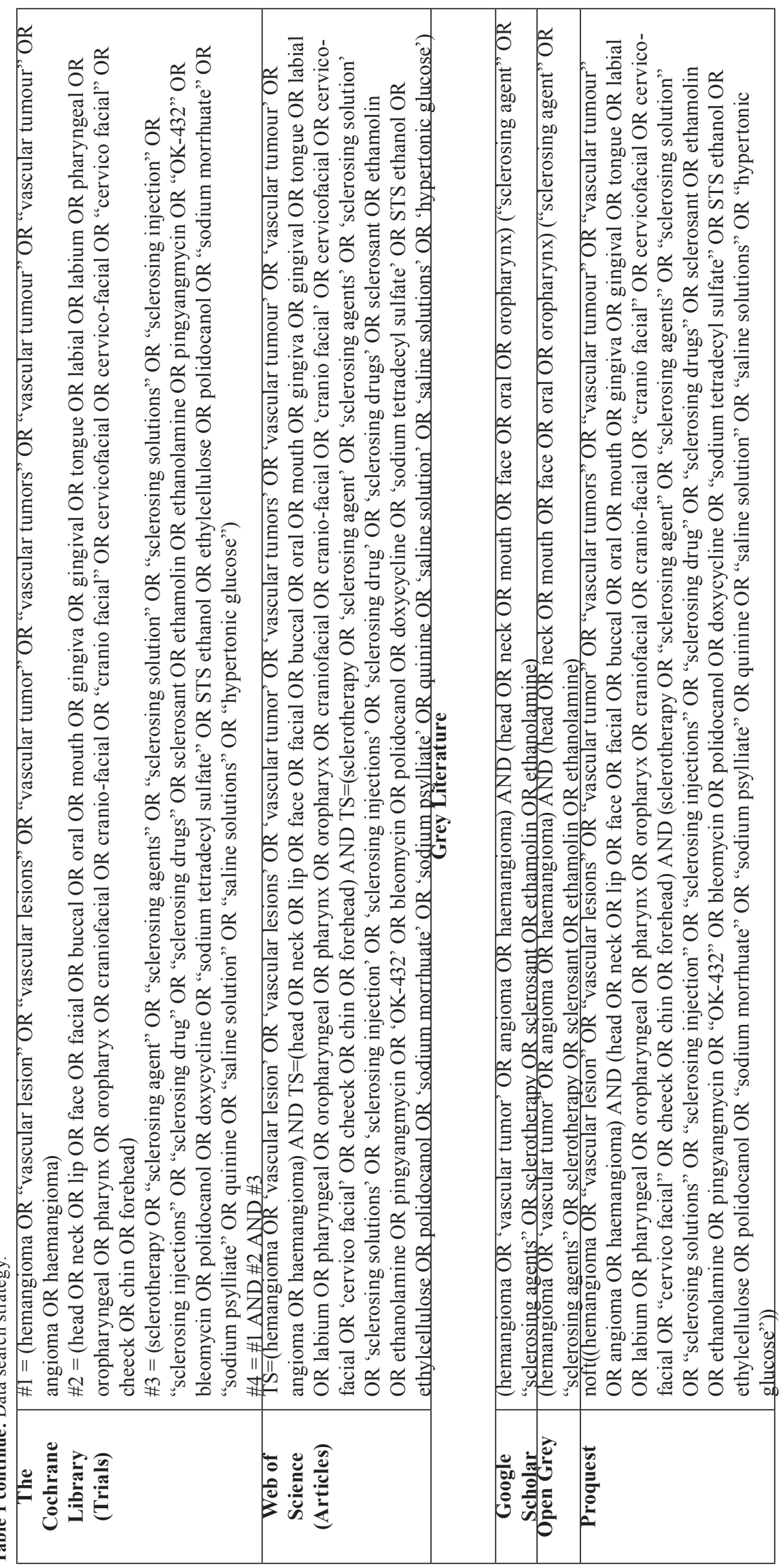


Institute Meta-Analysis of Statistics Assessment and Review Instrument (MAStARI), specific for descriptive studies. A third reviewer (G.M.) was involved in case of disagreements. Studies were categorized as "high" when reaching up to $49 \%$ score "yes"; "moderate" when reaching $50 \%$ to $69 \%$ score "yes"; and "low" when the study reached more than $70 \%$ score "yes".

Lesion size reduction and occurrence of collateral effects related to sclerotherapy (e.g. pain, ulceration, fever, hypo or hyperpigmentation, and others) were evaluated by means of absolute or relative differences between ba- seline and follow-up evaluations. In order to standardize results, values were described in percentage.

\section{Results}

The search strategy, after removing duplicates, resulted in 1239 records. Following title and abstract screening, fifty-six articles were considered eligible for full-text reading, of which 51 were excluded with reasons accordantly to eligibility criteria (Table 2, 2 continue). Thereafter, five studies were included for qualitative analysis (Fig. 1).

Table 2: Articles excluded and the reasons for exclusion $(n=51)$.

\begin{tabular}{|c|c|c|c|}
\hline Reference & \multicolumn{2}{|c|}{ Author (year) } & Reasons for Exclusion* \\
\hline 1. & Ali & 2016 & 4 \\
\hline 2. & Araújo & 2016 & 9 \\
\hline 3. & Baud & 2000 & 9 \\
\hline 4. & Carramaschi & 1991 & 9 \\
\hline 5. & Crawford & 2009 & 4 \\
\hline 6. & Fernandes & 2018 & 6 \\
\hline 7. & Fraulin & 2012 & 4 \\
\hline 8. & Gelbert & 2000 & 4 \\
\hline 9. & Govrin-Yehudain & 1987 & 6 \\
\hline 10. & Guo & 2014 & 8 \\
\hline 11. & Gupta & 2017 & 6 \\
\hline 12. & Han & 2016 & 8 \\
\hline 13. & Hassan & 2013 & 6 \\
\hline 14. & $\mathrm{He}$ & 2007 & 8 \\
\hline 15. & Hintringer & 2009 & 3 \\
\hline 16. & Hiraoka & 2012 & 6 \\
\hline 17. & Hou & 2008 & 8 \\
\hline 18. & Huang & 2006 & 8 \\
\hline 19. & Jianhong & 2005 & 3 \\
\hline 20. & Jiménez & 2009 & 6 \\
\hline 21. & Johann & 2005 & 6 \\
\hline 22. & Kane & 1995 & 6 \\
\hline 23. & Lameiro & 2018 & 2 \\
\hline 24. & Liu GJ & 2013 & 8 \\
\hline 25. & Liu SH & 1980 & 8 \\
\hline 26. & Liu XJ & 2009 & 4 \\
\hline 27. & Liu XJ & 2001 & 8 \\
\hline 28. & Luo & 2011 & 3 \\
\hline 29. & Muir & 2004 & 6 \\
\hline 30. & Pandey & 2018 & 3 \\
\hline 31. & Qin & 2002 & 8 \\
\hline 32. & Qin & 1995 & 8 \\
\hline 33. & Qiu & 2015 & 6 \\
\hline 34. & Ribeiro & 2015 & 6 \\
\hline
\end{tabular}


Table 2 continue: Articles excluded and the reasons for exclusion $(n=51)$.

\begin{tabular}{|l|c|c|c|}
\hline 35. & Sachin & 2013 & 6 \\
\hline $\mathbf{3 6 .}$ & Sainsbury & 2011 & 6 \\
\hline $\mathbf{3 7 .}$ & Saxena & 2013 & 9 \\
\hline $\mathbf{3 8 .}$ & Shou & 2000 & 8 \\
\hline $\mathbf{3 9 .}$ & Shou & 1996 & 8 \\
\hline $\mathbf{4 0 .}$ & Tai & 2015 & 2 \\
\hline $\mathbf{4 1 .}$ & Thakral & 2013 & 3 \\
\hline $\mathbf{4 2 .}$ & Thayal & 2012 & 8 \\
\hline $\mathbf{4 3 .}$ & Wang, C & 2000 & 8 \\
\hline $\mathbf{4 4 .}$ & Wang, L & 2009 & 9 \\
\hline $\mathbf{4 5 .}$ & Wang, Y & 2012 & 6 \\
\hline $\mathbf{4 6 .}$ & Winter & 2000 & 2 \\
\hline $\mathbf{4 7 .}$ & Woods & 1987 & 3 \\
\hline $\mathbf{4 8 .}$ & Yang & 2009 & 3 \\
\hline $\mathbf{4 9 .}$ & Zhang & 2016 & 8 \\
\hline $\mathbf{5 0 .}$ & Zheng & 1991 & 8 \\
\hline $\mathbf{5 1 .}$ & Zhou & 2002 & \\
\hline
\end{tabular}

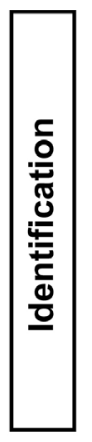

1580 records identified through database searching

Embase $=104$

LILACS $=50$

PubMed $=324$

SCOPUS $=754$

Web of Science $=337$
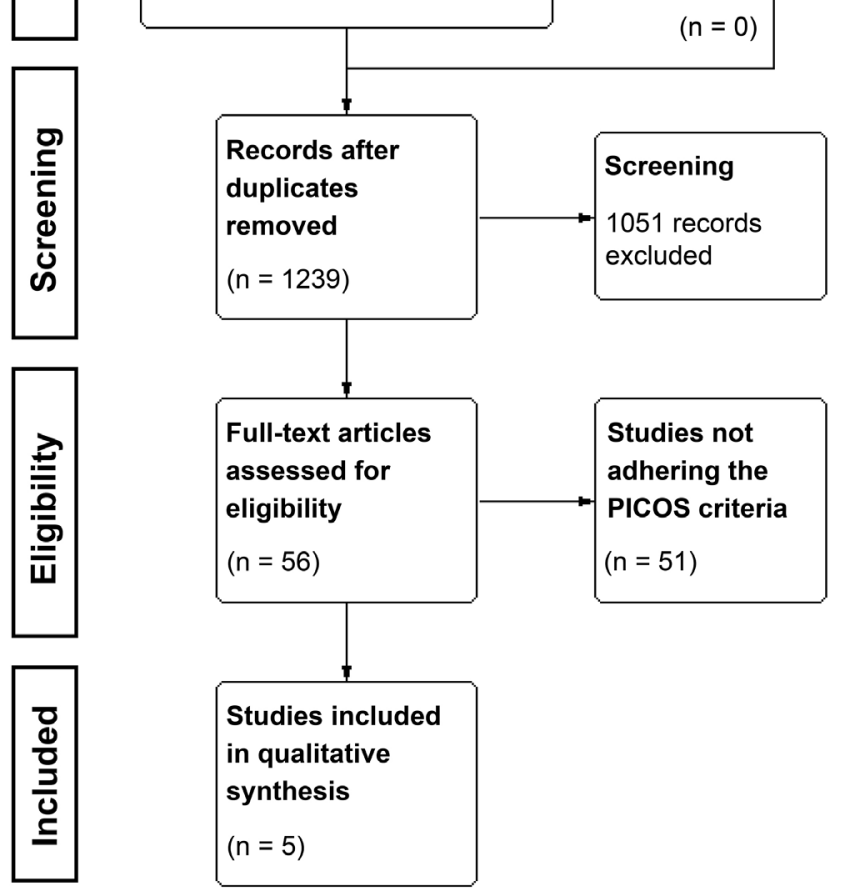

Fig. 1: Flow diagram of literature search and selection criteria (adapted from Preferred Reporting Items for Systematic Reviews and Meta-Analysis and generated using the software Review Manager 5.3, The Cochrane Collaboration). 
All included articles were descriptive studies, of which 4 were prospective case-series $(5,17-19)$ and 1 a retrospective study (20). One study was conducted in China (5), one in India (17), one in Brazil (20), one in South Africa (19), and 1 in Israel (18). Most studies were written in English (5,17-19) and 1 in Portuguese (20). Sample size ranged from 13 (20) to 66 (5) participants. Participants' age ranged from 2 months (5) to 79 years (18). Only 1 study did not provide any information considering adverse reactions (20). More information about study characteristics is available in Table 3,3 continue, 3 continue-1.

Overall, one study were judged at low, one at moderate, and 3 at high RoB. No included study investigated randomized or pseudo-random populations; however, this was expected since participants must present benign HN hemangiomas. Regarding confounders, three studies provided detailed description of methods $(5,17,19)$, whilst 2 did not provide sufficient information to permit a clear judgment $(18,20)$. Moreover, two studies used objective outcome measures, whilst others used subjective measures $(17,18,20)$. It should be noted that only 2 studies carried out an appropriate follow-up $(5,19)$. More information regarding RoB assessment is available in Figure 2.

-Results of individual studies

Agarwal et al. (2011) (17) investigated the effect of intralesional STS 3\% on hemangiomas of the tongue, lip, and palate in 20 participants (mean age not reported). Total regression was observed in $19(95 \%)$ individuals, whilst partial regression was observed in a single case $(5 \%)$. Pain and mild local inflammatory reaction were observed in all cases, sloughing and ulceration in 2 $(10 \%)$, and palatal perforation in 1 case $(5 \%)$.

Minkow et al. (1979) (18) studied the effect of STS 3\% on hemangiomas located in the lips, tongue, palate, and cheek mucosa in 24 participants (mean age 37.9 years). Overall, total remission was observed after a single application in small tumors $(0-1.5 \mathrm{~cm})$, whilst larger tumors $(2-4 \mathrm{~cm})$ required from 2 to 10 applications. No scars or defects were observed after treatment, however, most individuals (frequency not reported) presented pain and swelling and $2(8.3 \%)$ had pronounced inflammatory reaction.

Prado et al. (2011) (20) evaluated the therapeutic effect of intralesional EO (concentration not specified) on generic reported hemangiomas located in the lips, cheek, and tongue in 13 participants (mean age 62 years). Total regression was observed in all cases, although number of applications differed. A single application was required in $5(38.5 \%)$ participants, whilst $3(23.1 \%)$ required 2 applications, four (30.8\%) required 3 applications, and in 1 individual (7.7\%), four applications were required. Side effects were not reported in this study.

Pienaar et al. (2006) (19) assessed the effect of intrale- sional bleomycin $(0.3$ to $0.6 \mathrm{mg} / \mathrm{kg})$ on hemangiomas in 30 individuals (mean age 20 months). From these, twenty-six (86.7\%) individuals had lesions located in the cheeks, scalp, nose, lips, or eyelids. All individuals received 4 to 6 applications. Response-rates (rates higher than $90 \%$ were considered complete involution) regarding $\mathrm{HN}$ lesions were as follows: cheeks $(83 \%)$, scalp (90\%), nose (71\%), lips (86\%), and eyelids (90\%). It should be noted that adverse reactions were not separately reported for $\mathrm{HN}$ lesions and that 4 (13.3\%) individuals had lesions in other anatomical sites. Nevertheless, hyperpigmentation was observed in 13 (43.3\%) individuals, hypopigmentation in $5(16.7 \%)$, and scarring in 4 $(13.3 \%)$.

Hou et al. (2011) (5) investigated the effectiveness of intralesional pingyangmycin $(1 \mathrm{mg} / \mathrm{ml})$ on infantile hemangiomas of the forehead, nose, cheek, parotid/masseteric area, and lip in 66 individuals (mean age 5.6 months). The number of applications ranged from 1 to 6 and follow-up time from 1 to 4 years, occurring every 6 months. Complete cure of lesions was observed in 49 (74\%) individuals, whilst 9 (14\%) had substantial improvements, and $8(12 \%)$ showed mild improvements. Adverse reactions observed were local swelling (frequency not reported), fever lower than $38 \mathrm{oC}$ in individuals $14(21.2 \%)$, and anorexia in $8(12.1 \%)$.

-Synthesis of results

Clinical and methodological heterogeneity across studies were considered high. Since few studies were included and different substances and therapeutic protocols were observed, statistical pooling of data using meta-analysis was not considered appropriate.

Considering different sclerosing agents observed, two studies investigated STS 3\% $(17,18)$, one EO $(20)$, one assessed bleomycin A5 hydrochloride in the concentration of 0.3 to $0.6 \mathrm{mg} / \mathrm{kg}$ (19), and 1 study evaluated pingyangmycin $(1 \mathrm{mg} / \mathrm{ml})(5)$.

Most studies presented good results for sclerotherapy regarding lesion reduction. Total remission in all participants was reported by Prado et al. (2011) (20) and Minkow et al. (1979) (18), whilst total remission or major improvements were reported by Agarwal et al. (2011) (17), Hou et al. (2011) (5), and Pienaar et al. (2006) (19). The number of applications varied according to the size of lesion.

The following side-effects were reported across studies: pain $(17,18)$; swelling $(5,18)$; sloughing, ulceration, and palatal perforation (17); fever (5); anorexia (5); hyper/ hypopigmentation (19); scarring (19); and local inflammation $(17,18)$. The follow-up times were considerably discrepant, with a minimum follow-up of three months (19) and maximum of four years (5).

\section{Discussion}

Sclerotherapy on the management of hemangiomas has been a topic of interest in several studies as they 


\begin{tabular}{|c|c|c|c|}
\hline $\begin{array}{l}z \\
0 \\
0 \\
0 \\
0 \\
z \\
z \\
0 \\
0\end{array}$ & & 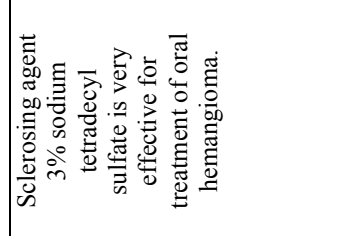 & 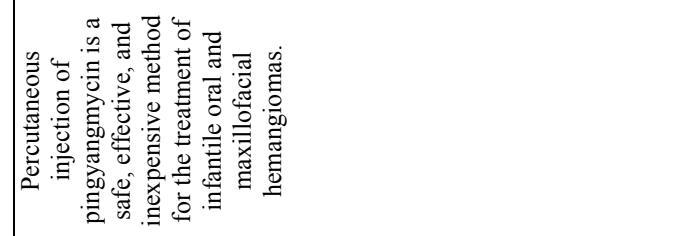 \\
\hline \multirow{2}{*}{ 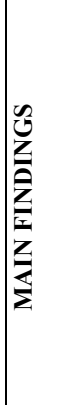 } & نे & 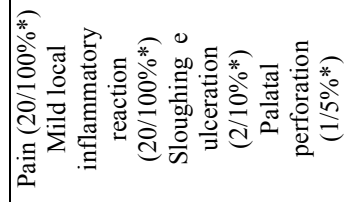 & 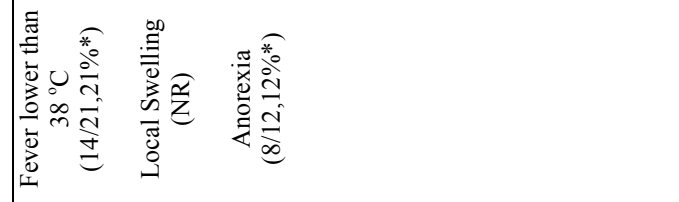 \\
\hline & 鿷 & 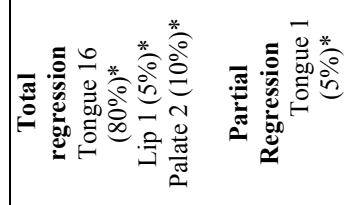 & 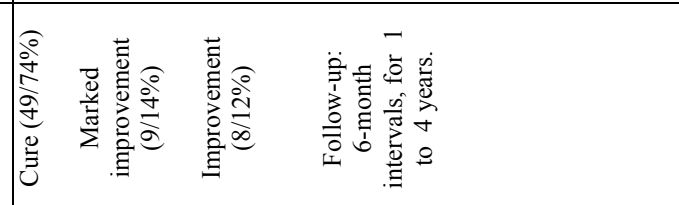 \\
\hline \multirow{3}{*}{ 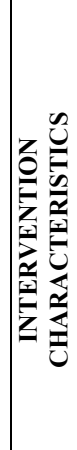 } & & 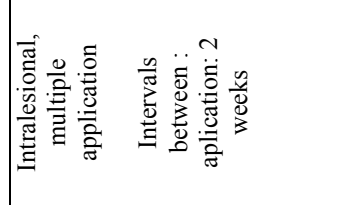 & 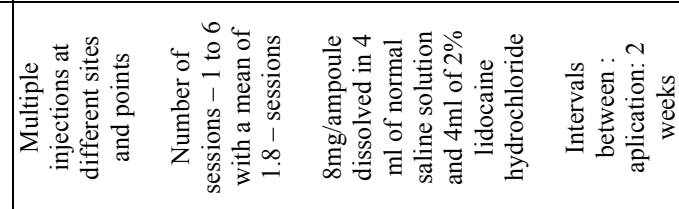 \\
\hline & $=$ & $\stackrel{\circ}{\circ}$ & - के छ \\
\hline & 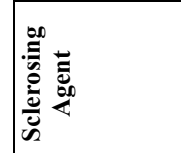 & 离 & 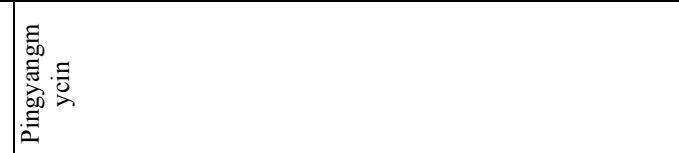 \\
\hline \multirow{5}{*}{ 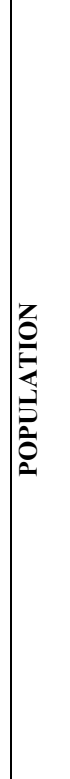 } & 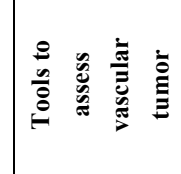 & 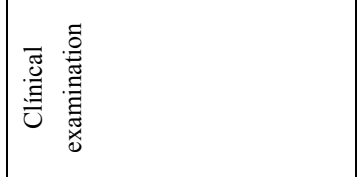 & 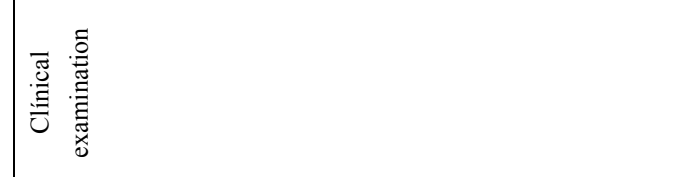 \\
\hline & 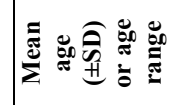 & ž & 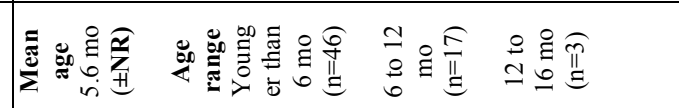 \\
\hline & 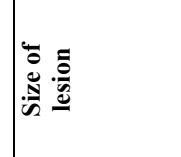 & $\tilde{z}$ & 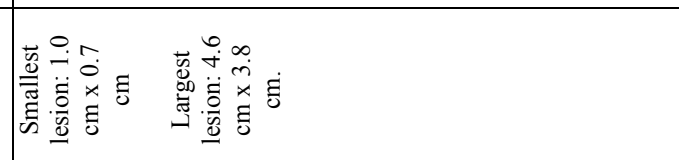 \\
\hline & 童高 & 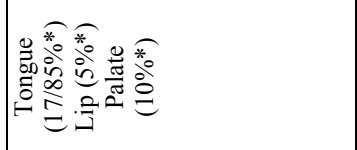 & 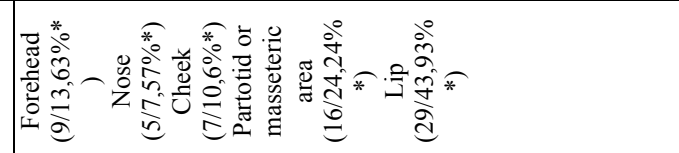 \\
\hline & 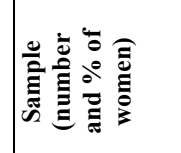 & $\frac{\widehat{a}}{z}$ & 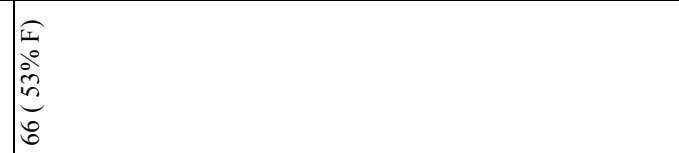 \\
\hline \multirow{2}{*}{ 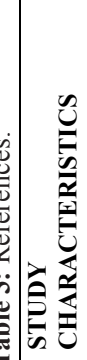 } & 竞 & 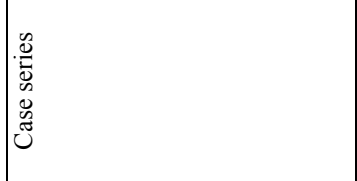 & 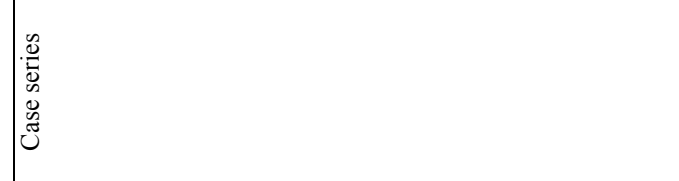 \\
\hline & 昰 & 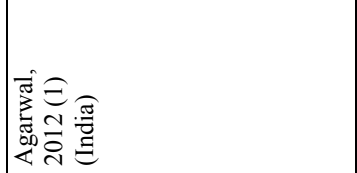 & 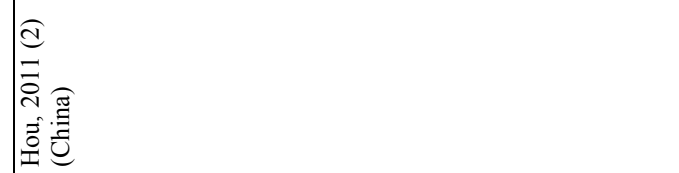 \\
\hline
\end{tabular}




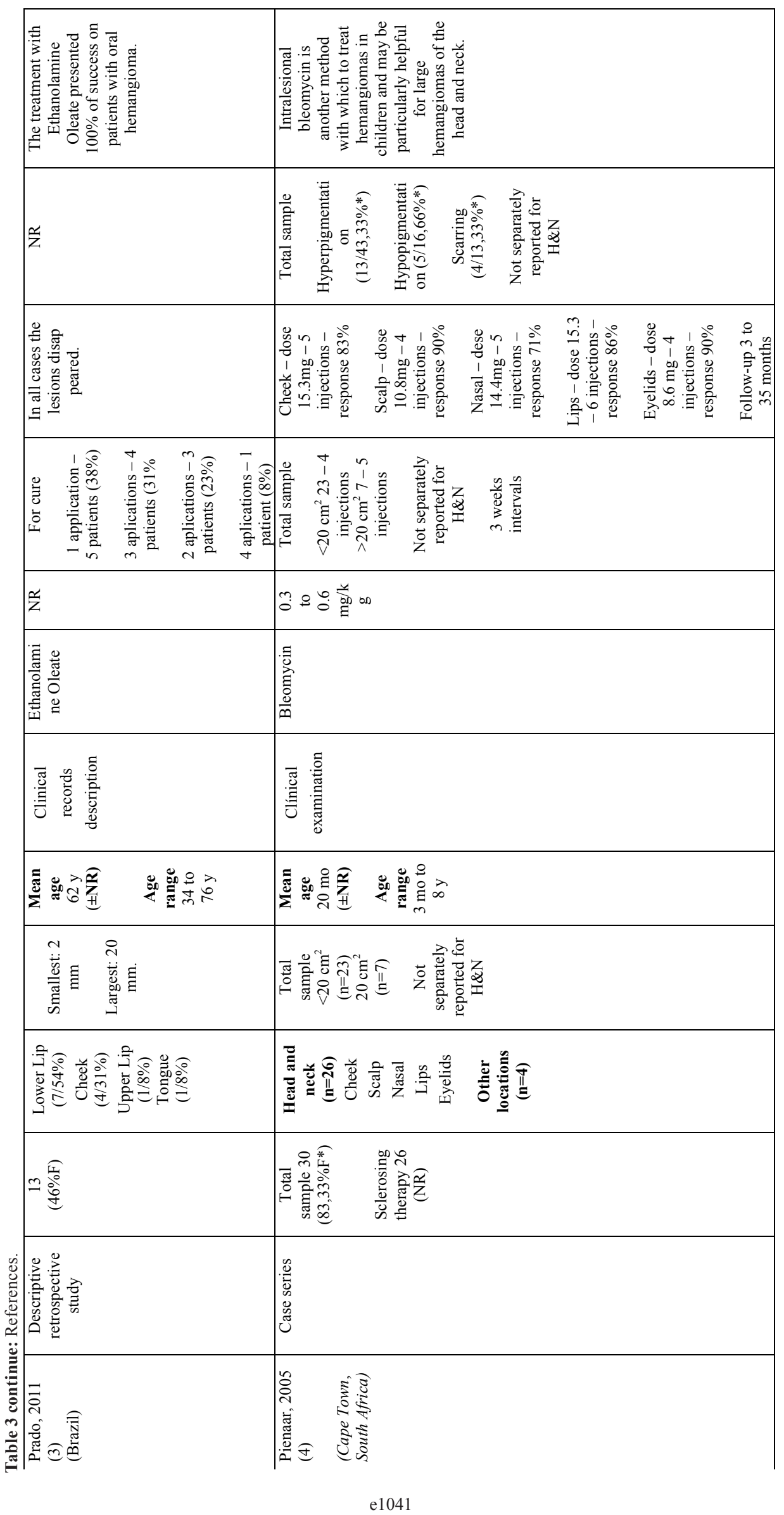




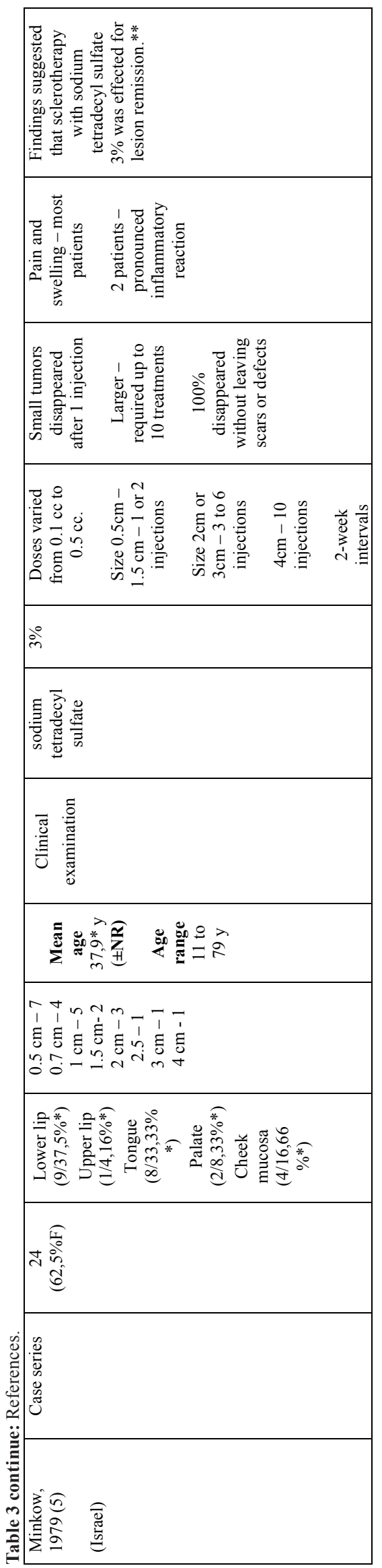

are considered a conservative method and are usually well tolerated. Still, the literature is sparse considering its effectiveness. This SR was performed to synthesize data gathered and guide clinical conducts and further researches regarding this topic. It was observed that, considering lesion remission, intralesional sclerotherapy presented overall good results with minor side effects. Nonetheless, evidence was considered weak and further controlled trials and objective outcome measurements are recommended.

In this SR, the studies of Agarwal et al. (2011) (17) and Minkow et al. (1979) (18) investigated STS 3\%, of which total remission was observed in all (18) or in the majority of participants (17). STS is detergent sclerosant that produce endothelial damage through multiple mechanisms leading to thrombosis and fibrosis $(21,22)$. This agent is considered to be less aggressive than absolute ethanol and has been used to treat $\mathrm{HN}$ vascular lesions (21).

Commonly reported adverse reactions in included studies investigating STS 3\% were pain, swelling, local inflammatory reaction, sloughing, ulceration and palatal perforation $(17,18)$. These findings are in accordance with current literature since swelling, pain, discoloration of the lesion, ulcer (23), and also fever and rashes (24) are common adverse reactions observed. The most reported side effect is tissue hyperpigmentation, although it is probably associated with use of inappropriately high concentrations or unexpectedly fragile veins. It should be highlighted that tissue necrosis has also been reported after administration of recommended doses, nonetheless, use of more dilute preparations of STS can lead to comparable results with decreased risk of necrosis (22). Furthermore, a single included study (20) evaluated intralesional sclerotherapy with EO and, although evidence was considered weak, total remission of the lesions were observed in all participants. EO is a detergent agent (21) that acts as sclerosant through endothelium damage, leading to thrombosis and fibrosis $(12,25)$. The effectiveness of EO is proposed to be similar to STS and safer compared to ethanol (21). No adverse effects were reported in the study of Prado et al. (2011) (20).

Still, since data were collected from charts, gaps in information about treatment outcomes might be present. Nonetheless, most commonly reported adverse effects of EO in current literature are redness, inflammation, pain during injection, tissue necrosis, and anaphylaxis (25). Although the study of da Silva et al. (2014) (12) was excluded due to insufficient number of participants, EO was used to treat oral hemangiomas and only a local burning sensation was reported as a side effect during administration.

Regarding other sclerosing agents, one study investigated the effects of bleomycin A5 hydrochloride (19). Bleomycin is a chemotherapeutic agent and, because of 


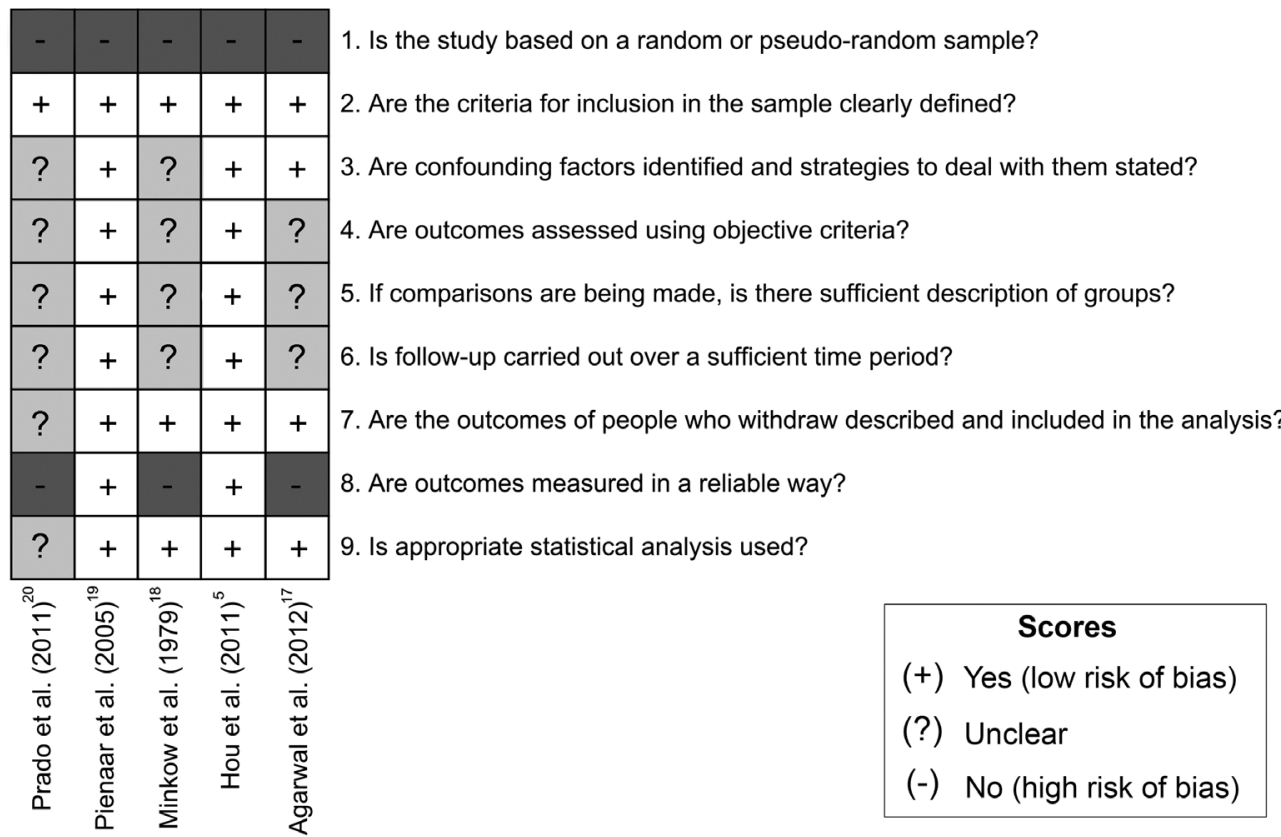

Fig. 2: Risk of bias summary, assessed by the Joanna Briggs Institute Meta-Analysis of Statistics Assessment and Review Instrument (MAStARI): author's judgments for each included study (generated using the software Review Manager 5.3, The Cochrane Collaboration).

its thrombogenicity capability, it has also been used in sclerotherapy (21). In the study of Pienaar et al. (2006) (19) bleomycin was used to treat hemangiomas. This agent showed good response-rates, including in lesions larger than $20 \mathrm{~cm}$.

The collateral effects reported were hyperpigmentation, hypopigmentation, and scarring. Known collateral effects caused by bleomycin A5 are edema, ulceration, scarring, nausea, lack of appetite (26) and tissue atrophy (27). Soft tissue atrophy is partly attributed to necrosis, although it might affect normal cells as well, and becomes more evident as the child grow up, since the tissue fails to grow (27). This collateral effect may be related to high dose or concentration of the sclerosing agent (27). A single included study (5) investigated Pingyangmycin to treat IH with maximum size of $4.6 \mathrm{~cm} \times 3.8 \mathrm{~cm}$, showing good results and few adverse reactions. Pyngyangmycin has a chemical structure similar to bleomycin, acting by damaging endothelial cells, which leads to collapse, shrinkage, and fibrosis of target tissues (27 68). There are many available options to treat HN hemangiomas, including surgery, systemic drugs (e.g. propanolol, atenolol, steroids), laser $(4,28)$, topical drugs (e.g. imiquimod), ultrasound (28), as well as sclerotherapy or just a follow-up (21), still there is not a gold standard. In cases of IH that are life-threatening, such as lesions obstructing the airway, systemic propranolol is considered the first choice of treatment (29). This method is also indicated in cases of existing or imminent functional impairment, ulceration, pain, and bleeding, as well as the risk of long-term or permanent disfigurement (30).
Propanolol is non-cardioselective blocker of beta-adrener $\neg$ gic receptors. It is proposed that propranolol inhibits vasodilation via beta-receptors, which decreases blood flow to the lesion; blocks the release of proangiogenic factors (e.g., VEGF, bFGF, MMP-2, and MMP-9), thus limiting the growth of $\mathrm{IH}$; and induces apoptosis in endothelial cells, favoring tumor remission (30).

Although propranolol is considered a conservative treatment, risk of systemic effects should be highlighted. The most common non-serious events related to oral propranolol are sleep disturbances, diarrhea, and constipation. Moreover, serious complications may occur, such as hypoglycemia or related seizure, bradycardia, hypotension, atrioventricular disturbances, and bronchospasm/ bronchial hyperreactivity (29). Due to potential systemic effects, topical administration has been suggested. A SR showed that good responses in size reduction with minimal side effects (itching and erythema) can be achieved, although treatment success might be related to longer treatment durations (31).

In this SR, primary studies were considerably heterogeneous, especially regarding methods. Since different sclerosing agents were used, no direct comparison could be performed. Moreover, results should be interpreted with caution, as the magnitude of observed effects might be overestimated due to lack of control groups. Therefore, athough overall good results were observed, further clinical studies with controlled design, standardized methods, and objective outcome measurements are recommended to better explore this topic.

Within the limitations of this SR, intralesional sclerothe- 
rapy with STS 3\%, EO, bleomycin, and pyngyamicin on the management of $\mathrm{HN}$ hemangiomas presented overall good results with minor adverse reactions, especially in regards to smaller lesions.

\section{References}

1. Dasgupta R, Fishman SJ. ISSVA classification. seminars in Pediatric surgery. Elsevier. 2014;23:158-161.

2. Avila ÉDd, Molon RSd, Conte Neto N, Gabrielli MAC, Hochuli-Vieira E. Lip cavernous hemangioma in a young child. Br Dent J. 2010;21:370-374.

3. Oksiuta M, Matuszczak E, Debek W, Dzienis-Koronkiewicz E, Hermanowicz A. Treatment of rapidly proliferating haemangiomas in newborns with propranolol and review of the literature. J Matern Fetal Neonatal Med. 2016;29:64-68.

4. Zheng JW, Zhang L, Zhou Q, Mai HM, Wang YA, Fan XD, et al. A practical guide to treatment of infantile hemangiomas of the head and neck. Int J Clin Exp Med. 2013;6:851.

5. Hou J, Wang M, Tang H, Wang Y, Huang H. Pingyangmycin sclerotherapy for infantile hemangiomas in oral and maxillofacial regions: an evaluation of 66 consecutive patients. Int J Oral Maxillofac Surg. 2011;40:1246-1251.

6. Mulliken JB, Enjolras O. Congenital hemangiomas and infantile hemangioma: missing links. J Am Acad Dermatol. 2004;50:875-882.

7. North PE. Classification and Pathology of Congenital and Perinatal Vascular Anomalies of the Head and Neck. Otolaryngol Clin North Am. 2018;51:1-39.

8. Adams DM, Ricci KW. Infantile Hemangiomas in the Head and Neck Region. Otolaryngol Clin North Am. 2018;51:77-87.

9. Enjolras O, Mulliken J. Vascular cutaneous anomalies in children: malformations and hemangiomas. Pediatr Surg Int. 1996;11:290-295. 10. Johann ACBR, Aguiar MCF, do Carmo MAV, Gomez RS, Castro WH, Mesquita RA. Sclerotherapy of benign oral vascular lesion with ethanolamine oleate: an open clinical trial with 30 lesions. Oral Surg Oral Med Oral Pathol Oral Radiol Endod. 2005;100:579-584.

11. Zweegers J, van der Vleuten CJ. The psychosocial impact of an infantile haemangioma on children and their parents. Arch Dis Child. 2012;97:922-6.

12. Da Silva WB, Ribeiro ALR, de Menezes SAF, Pinheiro JdJV, de Melo Alves-Junior S. Oral capillary hemangioma: A clinical protocol of diagnosis and treatment in adults. Oral Maxillofac Surg. 2014;18:431-437.

13. Horbach SE, Lokhorst MM, Saeed P, Rothová A, van der Horst CM. Sclerotherapy for low-flow vascular malformations of the head and neck: A systematic review of sclerosing agents. J Plast Reconstr Aesthet Surg. 2016;69:295-304.

14. Booth A, Clarke M, Ghersi D, Moher D, Petticrew M, Stewart L. An international registry of systematic-review protocols. Lancet. 2011;377:108-109.

15. Moher D, Liberati A, Tetzlaff J, Altman DG, Group P. Preferred reporting items for systematic reviews and meta-analyses: the PRISMA statement. Ann Intern Med. 2009;151:264-269, W264.

16. Greenhalgh T, Peacock R. Effectiveness and efficiency of search methods in systematic reviews of complex evidence: audit of primary sources. BMJ. 2005;331:1064-1065.

17. Agarwal S. Treatment of oral hemangioma with $3 \%$ sodium tetradecyl sulfate: study of 20 cases. Indian J Otolaryngol Head Neck Surg. 2012;64:205-207.

18. Minkow B, Laufer D, Gutman D. Treatment of oral hemangiomas with local sclerosing agents. Int J Oral Surg. 1979;8:18-21.

19. Pienaar C, Graham R, Geldenhuys S, Hudson DA. Intralesional bleomycin for the treatment of hemangiomas. Plast Recontr Surg. 2006;117:221-226.

20. Prado BN, dos Santos AF, Oliveira JMM, Vaccarezza GF, de Freitas CF. Uso de oleato de etanolamina para hemangiomas da cavidade bucal: um estudo de cinco anos. Revista de Odontologia da UNICID. 2011;23:42.

21. Heit JJ, Do HM, Prestigiacomo CJ, Delgado-Almandoz JA, Engli- sh J, Gandhi CD, et al. Guidelines and parameters: percutaneous sclerotherapy for the treatment of head and neck venous and lymphatic malformations. J Neurointerv Surg. 2017;9:611-617.

22. Duffy DM. Sclerosants: a comparative review. Dermatol Surg. 2010;36:1010-1025.

23. O'Donovan JC, Donaldson JS, Morello FP, Pensler JM, Vogelzang RL, Bauer B. Symptomatic hemangiomas and venous malformations in infants, children, and young adults: treatment with percutaneous injection of sodium tetradecyl sulfate. AJR Am J Roentgenol. 1997; 169:723-729.

24. Harjai MM, Jha M. Intralesional bleomycin and sodium tetradecyl sulphate for haemangiomas and lymphangiomas. Afr J Paediatr Surg. 2012;9.

25. do Monte Lameiro TM, Denadai R, Pereira-Filho JC, Raposo-Amaral CA, Raposo-Amaral CE. Treatment of Facial Infantile Hemangioma: Comparative Study Between Propranolol and Ethanolamine Oleate. J Craniofac Surg. 2018;29:1514-1517.

26. Luo Q-f, Zhao F-y. The effects of Bleomycin A5 on infantile maxillofacial haemangioma. Head Face Med. 2011;7:11.

27. Qiu Y, Lin X, Ma G, Chang L, Jin Y, Chen H, et al. Eighteen Cases of Soft Tissue Atrophy After Intralesional Bleomycin A5 Injections for the Treatment of Infantile Hemangiomas: A Long-Term Follow-Up. Pediatr Dermatol. 2015;32:188-191.

28. Novoa M, Baselga E, Beltran S, Giraldo L, Shahbaz A, Pardo-Hernandez $\mathrm{H}$, et al. Interventions for infantile haemangiomas of the skin. Cochrane Database Syst Rev. 2018.

29. Léaute-Labrèze C, Boccara O, Degrugillier-Chopinet C, Mazereeuw-Hautier J, Prey S, Lebbé G, et al. Safety of oral propranolol for the treatment of infantile hemangioma: a systematic review. Pediatrics. 2016;2016:e20160353.

30. Rotter A, de Oliveira ZNP. Infantile hemangioma: pathogenesis and mechanisms of action of propranolol. J Dtsch Dermatol Ges. 2017;15:1185-1190.

31. Price A, Rai S, Mcleod R, Birchall J, Elhassan H. Topical propranolol for infantile haemangiomas: A systematic review. J Eur Acad Dermatol Venereol. 2018;32:2083-2089.

\section{Acknowledgements}

Rúbia T. Stuepp [number 201705960], Fernanda M. Scotti [number 1740542], and Gilberto Melo [number 88887.200723/2018-00] are supported with scholarships by CAPES/FAPESC (Coordination for the Improvement of Higher Education Personnel/Foundation for the Support of Research and Innovation in the State of Santa Catarina), Ministry of Education, Brazil.

\section{Conflict of interest}

The authors have no conflicts of interest to declare. 